\title{
Preferência alimentar, efeito da planta hospedeira e da densidade larval na sobrevivência e desenvolvimento de Dione juno juno (Cramer) (Lepidoptera, Nymphalidae) ${ }^{1}$
}

\author{
Vidica Bianchi ${ }^{2} \&$ Gilson R. P. Moreira ${ }^{2}$ \\ ${ }^{1}$ Contribuição número 449 do Departamento de Zoologia, Universidade Federal do Rio Grande do Sul. \\ 2 Departamento de Zoologia, Instituto de Biociências, Universidade Federal do Rio Grande do Sul. Avenida Bento Gonçalves \\ 9500,91501-970 Porto Alegre, Rio Grande do Sul, Brasil.E-mail vidicab@zipmail.com.br, gilson.moreira@ufrgs.br
}

\begin{abstract}
Feeding preference, host-plant and larval density effects on survivorship and growth rates of Dione juno juno (Cramer) (Lepidoptera, Nymphalidae). Ten passion vine species from Rio Grande do Sul were evaluated regarding larval feeding preference and performance of Dione juno juno (Cramer, 1779): Passifora alata Dryander, 1781; P. amethystina Mikan, 1820; P. caerulea Linnaeus, 1753; P. capsularis Linnaeus, 1753; P. edulis Sims, 1818; . elegans Masters, 1872; P. misera Humbold, Bonpland et Kunth, 1817; P. suberosa Linnaeus, 1753; P. tenuifila Killip, 1927 and $P$. warmingii Masters, 1872. Larval density effect on performance was also tested on $P$. edulis, by using seven larval group sizes: one, two, eight, sixteen, thirty-two and sixty-four larvae. Larval feeding preferences were evaluated through leaf disk, single and multiple choice tests. Larvae achieved the greatest survivorship on $P$. misera, $P$. tenuifila and $P$. edulis. None survived on P. alata, P. capsularis, $P$. amesthystina, $P$. suberosa, and $P$. warmingii. Larvae chose $P$. edulis on multiple choice tests. They ingested similar amounts of $P$. tenuifila, $P$. misera and $P$. caerulea on single choice tests. Larval growth rates were greater, and adults were larger when reared on $P$. misera compared to $P$. edulis. Survivorship was significantly reduced on group sizes of one, two and four larvae, and thus may account for the larval aggregation behavior on this species. We concluded that a few passion vine species other than $P$. edulis can act as potential hostplants for D. juno juno in Rio Grande do Sul State. From an ecological perspective, however, most of these alternative host-plant species present limitations regarding either suitability, plant size or abundance.

KEY WORDS. Heliconian butterflies, host-plant selection, insect herbivory, passion vines.
\end{abstract}

RESUMO. Dez espécies de passifloraceas ocorrentes no Rio Grande do Sul foram avaliadas em relação à preferência alimentar e performance larval de Dione juno juno (Cramer, 1779) (Lepidoptera, Nymphalidae): Passifora alata Dryander, 1781; P. amethystina Mikan, 1820; P. caerulea Linnaeus, 1753; P. capsularis Linnaeus, 1753; P. edulis Sims, 1818; P. elegans Masters, 1872; P. misera Humbold, Bonpland et Kunth, 1817; P. suberosa Linnaeus, 1753; P. tenuifila Killip, 1927 e $P$. warmingii Masters, 1872. O efeito da densidade larval na performance foi também testado em $P$. edulis: grupos de uma, duas, quatro, oito, dezesseis, trinta e duas, e sessenta e quatro larvas. A preferência das larvas foi avaliada com base em teste utilizando-se discos foliares, com e sem chance de escolha. As larvas obtiveram maior sobrevivência em $P$. misera, $P$. tenuifila e $P$. edulis. Nenhuma sobreviveu em P. alata, $P$. capsularis, $P$. amesthystina, $P$. suberosa e $P$. warmingii. As larvas escolheram $P$. edulis nos testes com chance de escolha. Ingeriram quantidades semelhantes de $P$. tenuifila, $P$. misera e $P$. caerulea nos testes sem chance de escolha. A taxa de crescimento larval e o tamanho dos adultos foi maior quando criadas em P. misera, quando comparado com P. edulis. A sobrevivência foi significativamente reduzida nos grupos com uma, duas e quatro larvas, o que pode explicar em parte o comportamento gregário desta espécie. Concluiuse que poucas espécies de passifloráceas além de $P$. edulis podem constituir-se em hospedeiras potenciais de $D$. juno juno no Estado do Rio Grande do Sul. Numa perspectiva ecológica, no entanto, muitas destas hospedeiras alternativas apresentam limitações a respeito de sua adequabilidade, tamanho ou abundância da planta.

PALAVRAS CHAVE. Heliconíneos, herbivoria, passifloráceas, seleção de plantas hospedeiras.

A busca de alimento pode levar ao uso de diferentes recursos
nutricionais, e estes por sua vez, podem conferir-lhes varia-
ções no valor adaptativo, dependendo de seu efeito sobre os
componentes da história de vida dos animais. Assim, o desem- penho de um dado animal depende da dieta, tanto em quantidade quanto em qualidade. Uma estratégia de forrageamento adequado é aquela que maximiza os ganhos nutricionais provenientes da alimentação e minimiza os riscos de mortalidade. 
Dessa forma, principalmente no caso dos insetos holometábolos, os quais maximizam o desenvolvimento somático nos estágios imaturos, larvas alimentadas adequadamente podem originar adultos com maior capacidade de movimentação, competição, defesa e reprodução (SLANSKY 1993).

Os heliconíneos (Lepidoptera, Nymphalidae) utilizam plantas da família Passifloraceae para oviposição e alimentação de suas larvas. Estes dois grupos de organismos encontramse amplamente distribuídos na região Neotropical (Benson et al. 1976). O desempenho dos heliconíneos pode estar relacionado ao tipo de planta hospedeira utilizada. Por exemplo, Heliconius erato phyllis (Fabricius, 1775) utiliza pelo menos oito espécies de passifloráceas no Rio Grande do Sul, as quais conferem às larvas diferentes taxas de desenvolvimento (MennaBarreto \& Araújo 1985, Périco 1995). Dentre essas, Rodrigues \& Moreira $(2002,2004)$ demonstraram que as fêmeas de $H$. erato phyllis provenientes de larvas alimentadas com Passiflora misera apresentam maior tamanho e maior fecundidade em relação aquelas criadas em Passiflora suberosa, utilizando em campo predominantemente a primeira, conforme a abundância correspondente. Em adição, foi constatado para esse heliconíneo uma preferência inata por $P$. misera, quer como alimento para as larvas como substrato para oviposição quando adulto (KERPEL \& Moreira, no prelo). Estudos intensivos, com este enfoque, considerados básicos do ponto de vista da interação insetoplanta, são inexistentes para a maioria das demais espécies de heliconíneos que ocorrem no sul do Brasil.

No Estado do Rio Grande do Sul (RS) ocorrem 14 espécies de passifloráceas (SACCO 1980). Entre elas são registradas como hospedeiras de Dione juno juno (Cramer): Passiflora actinia Hooker, 1943 (Dell'Erba et al. no prelo), P. caerulea (Biezanko 1949, Brown \& Mielke 1972), P. capsularis, P. edulis, P. misera e P. tenuifila (G. L. Garcias, dados não publicados) e além dessas, também $P$. elegans, conforme observações adicionais. P. edulis, em particular, é utilizada mundialmente em plantios comerciais de maracujá (VANDERPLank 1991). Por usar com frequência esta espécie e causar-lhe severas desfolhas, D. juno juno é considerada praga dos pomares comerciais de maracujá (LARA et al. 1999).

O papel das diferentes espécies de passifloráceas que ocorrem no RS frente ao desempenho e preferência de $D$. juno juno é desconhecido, exceto em relação a estudos correspondentes realizados em outros locais com respeito a $P$. alata, $P$. edulis e $P$. actinia (BoIçA JR. et al. 1999). Tais informações são importantes não somente do ponto de vista da ecologia evolutiva dessa espécie, mas também em relação às estratégias de manejo em pomares comerciais no Sul do Brasil, no que tange ao uso de plantas hospedeiras alternativas.

Dione juno coloca os ovos em massa (60 a 140 ovos), na face abaxial das folhas, e as larvas apresentam comportamento gregário (LoRdello 1956, MuYshondt et al. 1973, BROWN 1981, G.L. Garcias, dados não publicados). Assim, além da espécie de planta hospedeira, a agregação larval também pode afetar seu desempenho, como por exemplo, limitando a disponibilidade de alimento em plantas de pequeno porte. Tal comportamento pode proporcionar vantagens, incluindo um forrageio mais eficiente, maior capacidade de defesa contra inimigos naturais e termorregulação (Fitzgerald 1993, Clark \& Faeth 1997, Denno \& BenRey 1997, BRyANT et al. 2000), o que ainda necessita ser explorado em relação a $D$. juno juno.

O presente trabalho objetiva estabelecer bases para estudos quanto à ecologia da interação de $D$. juno juno com as espécies de passifloráceas hospedeiras e o papel correspondente ao comportamento gregário das larvas, bem como fornecer subsídios para o manejo da espécie em pomares comerciais. Especificamente, investiga-se o efeito da planta hospedeira e da densidade larval na taxa de desenvolvimento e sobrevivência das larvas, bem como no tamanho dos adultos. Avalia-se também a preferência alimentar das larvas em relação às cinco espécies de maracujás ocorrentes no RS.

\section{MATERIAL E MÉTODOS}

Para avaliar o efeito da planta hospedeira no desenvolvimento, foram coletados ovos em P. edulis f. flavicarpa Deg., cultivados na região de Porto Alegre e Eldorado do Sul, RS. Estes foram acondicionados em placas de Petri, forradas com papel filtro umedecido, que foram mantidas em câmara climatizada ( 14 horas luz/dia, $75 \pm 5 \%$ UR e $25 \pm 1^{\circ} \mathrm{C}$ ) até a eclosão. Tanto os ovos quanto os ramos de $P$. edulis utilizados nos experimentos foram tratados com solução aquosa de hipoclorito de sódio (1\%), para evitar contaminações por patógenos, observados nas populações de $D$. juno juno no Brasil (ANDRAde \& Habib 1984).

Após a eclosão, as larvas, em grupos de três, foram transferidas para ramos das diferentes espécies de passifloráceas (20 ramos por espécies): P. alata, $P$. amethystina, $P$. caerulea, $P$. capsularis, $P$. edulis f. flavicarpa, P. elegans, $P$. misera, $P$. suberosa, $P$. tenuifila e $P$. warmingii. Os ramos foram coletados nos arredores de Porto Alegre e acondicionados em garrafas plásticas de água mineral $(500 \mathrm{ml})$, providas de armação de arame e bambu e cobertura de filó (Mugrabi-Oliveira \& Moreira 1996), e mantidas em câmara climatizada.

Larvas foram observadas diariamente para a reposição do alimento, quantificação da sobrevivência, da duração dos cinco ínstares larvais e da duração do estágio pupal. Para identificar as ecdises, foi utilizado tinta atóxica de cores diferentes $\left(\right.$ Acrilex $\left.{ }^{\circledast}\right)$, na região dorso-posterior do penúltimo segmento abdominal (TAVAres et al. 2002). Para medir o tamanho da asa anterior dos adultos foi utilizado um paquímetro (RoDRIGUES \& MoReira 2002).

Para avaliar a preferência alimentar frente as espécies que permitiram sobrevivência, foram realizados testes com e sem possibilidade de escolha (LewIS \& VAN EMDEN 1986), utilizandose larvas adicionais, criadas em $P$. edulis. Estas foram submetidas aos testes no primeiro e no quinto ínstar (20 repetições cada caso). Nos testes com chance de escolha, foram oferecidos discos foliares de P. caerulea, P. edulis, P.elegans, P. misera e $P$. 
tenuifila em conjunto, e naqueles sem chance de escolha, foram oferecidos discos foliares de apenas uma espécie de passiflorácea. Discos foliares foram obtidos através de um vazador (diâmetro $=95 \mathrm{~mm}^{2}$ ) e posicionados de forma equidistante e intercalados, dentro de pote plástico sobre papel filtro umedecido, presos por alfinetes entomológicos (Hanson 1983). A área oferecida foi próxima ao dobro do consumo médio para cada ínstar durante um período de cinco horas, conforme descrito em Rodrigues \& MoreIra (1999). Larvas de primeiro ínstar foram testadas em grupos de cinco e, as de quinto, individualmente. No início de cada teste, foram removidas do sistema de criação com o auxílio de um pincel e colocadas no centro do pote. Este foi coberto com um filme transparente de polietileno. Restos dos discos foliares foram colados em papel e posteriormente determinada a área consumida; medida em mesa com luz refletida, através da sobreposição dos restos dos discos foliares em papel milimetrado (Rodrigues \& Moreira 1999).

Para avaliar o efeito da densidade larval no desenvolvimento, larvas recém-eclodidas foram transferidas para ramos de $P$. edulis e testadas em sete diferentes densidades: grupos de uma $(n=20)$, duas $(n=20)$, quatro $(n=20)$, oito $(n=10)$, dezesseis $(n=10)$, trinta e duas $(n=10)$ e sessenta e quatro larvas $(n=5)$. O procedimento para a criação das larvas foi o mesmo adotado para avaliar o efeito das plantas hospedeiras.

Os dados referentes a sobrevivência foram organizados em tabelas de contingências e comparados através do Teste Exato de Fisher, seguido pela correção de Bonferroni. A distribuição dos dados de tempo de desenvolvimento, tamanho dos adultos e preferência alimentar foram avaliados quanto à normalidade pelo teste de Kolmogorov-Smirnov e quanto à homocedasticidade pelo teste de Bartlett. Os dados referentes ao tempo de desenvolvimento, tamanho dos adultos e a preferência alimentar nos testes sem chance de escolha com larvas de quinto ínstar, foram submetidos à uma Anova (um fator), seguida de testes múltiplos de Tukey. Dados referentes ao consumo foliar nos testes com chance de escolha, no primeiro e no quinto ínstar, foram transformados $[\log (\mathrm{n}+1)]$ para a análise de variância. Aqueles referentes aos testes de preferência alimentar sem chance de escolha com larvas de primeiro ínstar, não passaram nos testes de normalidade, mesmo após a transformação. Desta forma, foram comparados pelo teste não-paramétrico de KruskalWallis, seguidos por testes múltiplos de Dunn. Os testes paramétricos e não-paramétricos seguiram os critérios descritos por Zar (1999) e Conover (1980), respectivamente.

\section{RESULTADOS}

Das dez espécies de passifloráceas oferecidas, D. juno juno sobreviveu de forma variada em cinco. A maior sobrevivência foi em P. misera, P. tenuifila e P. edulis (Fig. 1). A sobrevivência foi desprezível em $P$. elegans, não sendo diferente estatisticamente de $P$. alata, $P$. amethystina, $P$. capsularis, $P$. suberosa e $P$. warmingi, sobre as quais nenhuma larva completou o desenvolvimento.

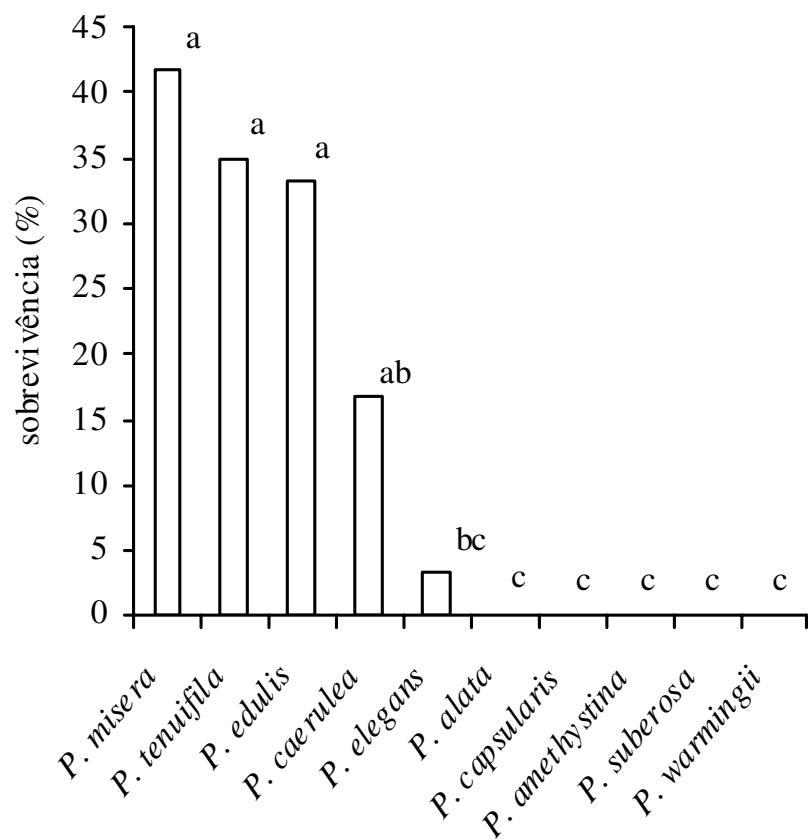

Figura 1. Sobrevivência de $D$. juno juno, em diferente passifloráceas. Colunas seguidas de letras distintas diferem significativamente (testes exatos de Fisher, com correção de Bonferroni, $\alpha=0,0033$ ).

Levando-se em conta apenas as espécies de passifloráceas onde a sobrevivência foi mais alta, a menor sobrevivência ocorreu no primeiro ínstar em todos os casos (Fig. 2).

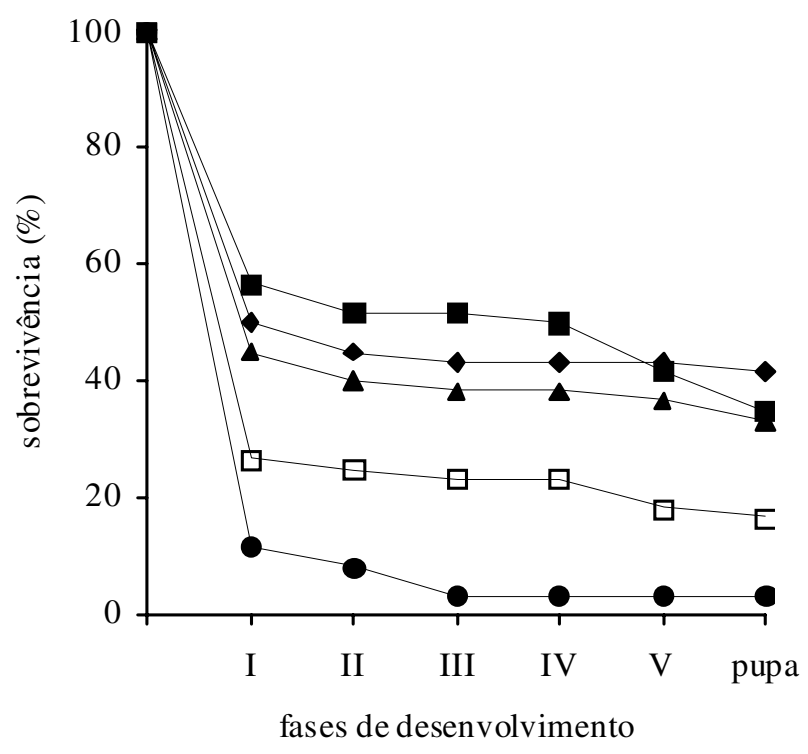

Figura 2. Sobrevivência dos imaturos de $D$. juno juno ao longo das fases de desenvolvimento, quando alimentados com diferentes passifloráceas. Os números romanos representam os ínstares larvais.

Revista Brasileira de Zoologia 22 (1): 43-50, março 2005 
Dentre as espécies onde houve sobrevivência expressiva, o tempo de desenvolvimento foi menor em $P$. misera do que em $P$. edulis, as quais não diferiram de $P$. tenuifila e $P$. caerulea, que não diferiram entre si (Fig. 3).

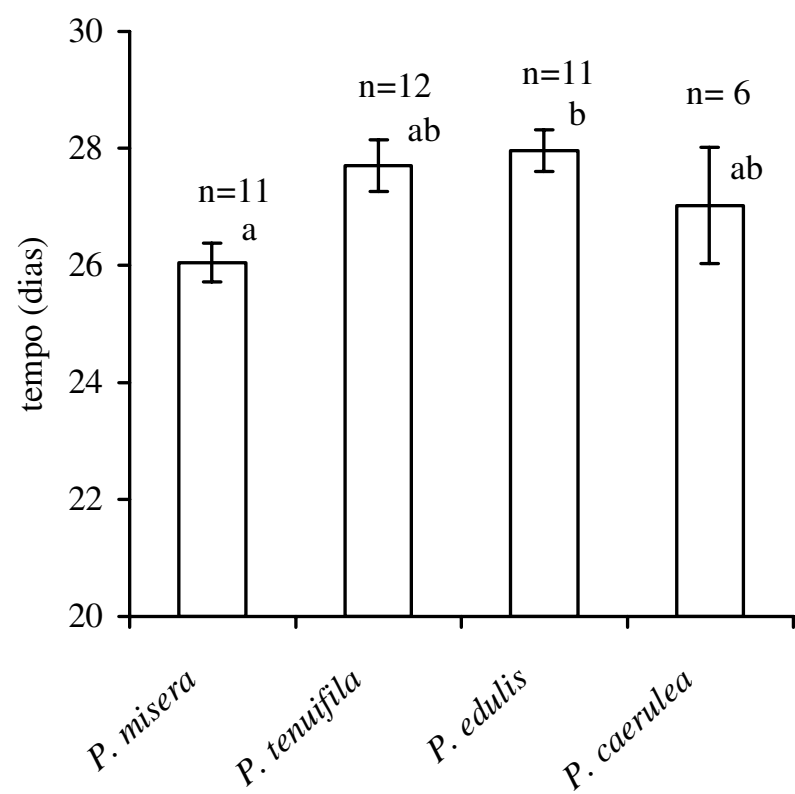

Figura 3. Tempo de desenvolvimento (média \pm erro padrão) dos imaturos de $D$. juno juno, quando criados em diferentes espécies de passifloráceas. Colunas seguidas de letras distintas diferem significativamente. (Anova, um fator, $F=3,475, p=0,0258$; testes múltiplos de Tukey, $\alpha=0,05)$.

O tamanho dos adultos foi maior em P. misera e P. edulis, sendo que nesta última não diferiu de $P$. tenuifila. Os adultos provenientes das larvas alimentadas com $P$. caerulea foram significativamente menores quando comparado com os demais (Fig. 4). Do total de 21 indivíduos que emergiram em P. tenuifila, $8(38,1 \%)$ apresentaram asas deformadas, o que não ocorreu nas demais.

Nos testes com chance de escolha, as larvas de primeiro ínstar preferiram $P$. edulis em relação a $P$. caerulea e $P$. elegans, entre as quais não houve diferença significativa (Fig. 5). Neste tipo de teste, as larvas de quinto ínstar preferiram $P$. edulis e $P$. caerulea, quando comparadas com P. misera e P. elegans (Fig. 6).

Nos testes sem chance de escolha, as larvas de primeiro ínstar consumiram maior área foliar em $P$. edulis, $P$. tenuifila e $P$. misera do que em P. elegans (Fig. 7). Em relação ao quinto ínstar, neste teste, utilizaram mais $P$. edulis que $P$. elegans (Fig. 8).

A sobrevivência foi significativamente menor nos tratamento com uma, duas e quatro larvas, em relação aqueles com oito, dezesseis, trinta e duas, e sessenta e quatro larvas (Fig. 9).

Com relação ao tempo de desenvolvimento em diferen-

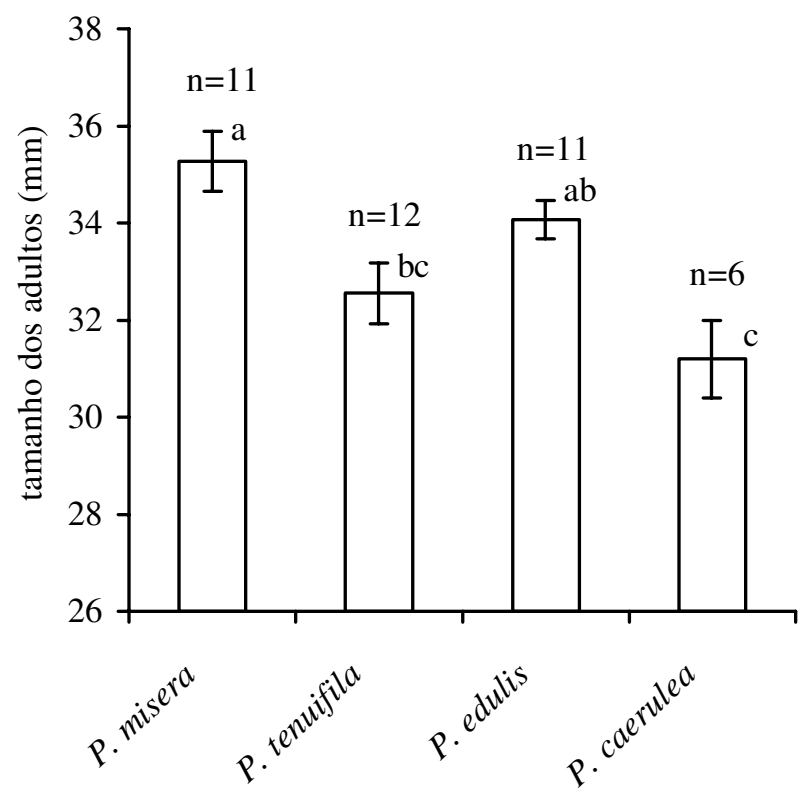

Figura 4. Tamanho dos adultos (média \pm erro padrão) de $D$. juno juno, quando criados em diferentes passifloráceas. Colunas seguidas de letras distintas diferem significativamente (Anova, um fator, $\mathrm{F}=7,862, \mathrm{p}=0,0001$; testes múltiplos de Tukey, $\alpha=0,05$ )

tes densidades, ocorreu diferença significativa entre os tratamentos com uma e trinta e duas larvas (Fig. 10). Quanto ao tamanho dos adultos, as diferenças correspondentes não foram significativas (Fig. 11).

\section{DISCUSSÃO}

Os dados demonstraram claramente que a espécie de planta hospedeira e a densidade larval afetam a sobrevivência da espécie. Diversas das espécies de passifloráceas que ocorrerem no RS inviabilizam o desenvolvimento deste lepidoptero, e as que lhe permitiram desenvolvimento interferem de forma diferenciada no tempo de desenvolvimento e tamanho dos adultos. Foi demonstrado também, que a preferência da larva nem sempre é pela planta que lhe confere melhor desempenho.

De certa forma, a habilidade de D. juno juno desenvolverse com sucesso em quatro espécies de passifloráceas pode indicar inicialmente que ela é mais generalista quando comparada com outros heliconíneos, como por exemplo, aqueles pertencentes ao anel mimético "laranja", que ocorrem no Parque do Turvo (G. L. Garcias, dados não publicados). Diversos estudos tem demonstrado, entretanto, que com poucas exceções (por exemplo, Dione moneta moneta Hübner, 1825, encontrada exclusivamente sobre $P$. warmingii), o uso ocasional de mais de uma espécie de passiflorácea é a regra para o estágio larval dos heliconíneos no RS (Dell'Erba et al., no prelo). A utilização de várias espécies de hospedeiras pode lhes permitir maior suces- 

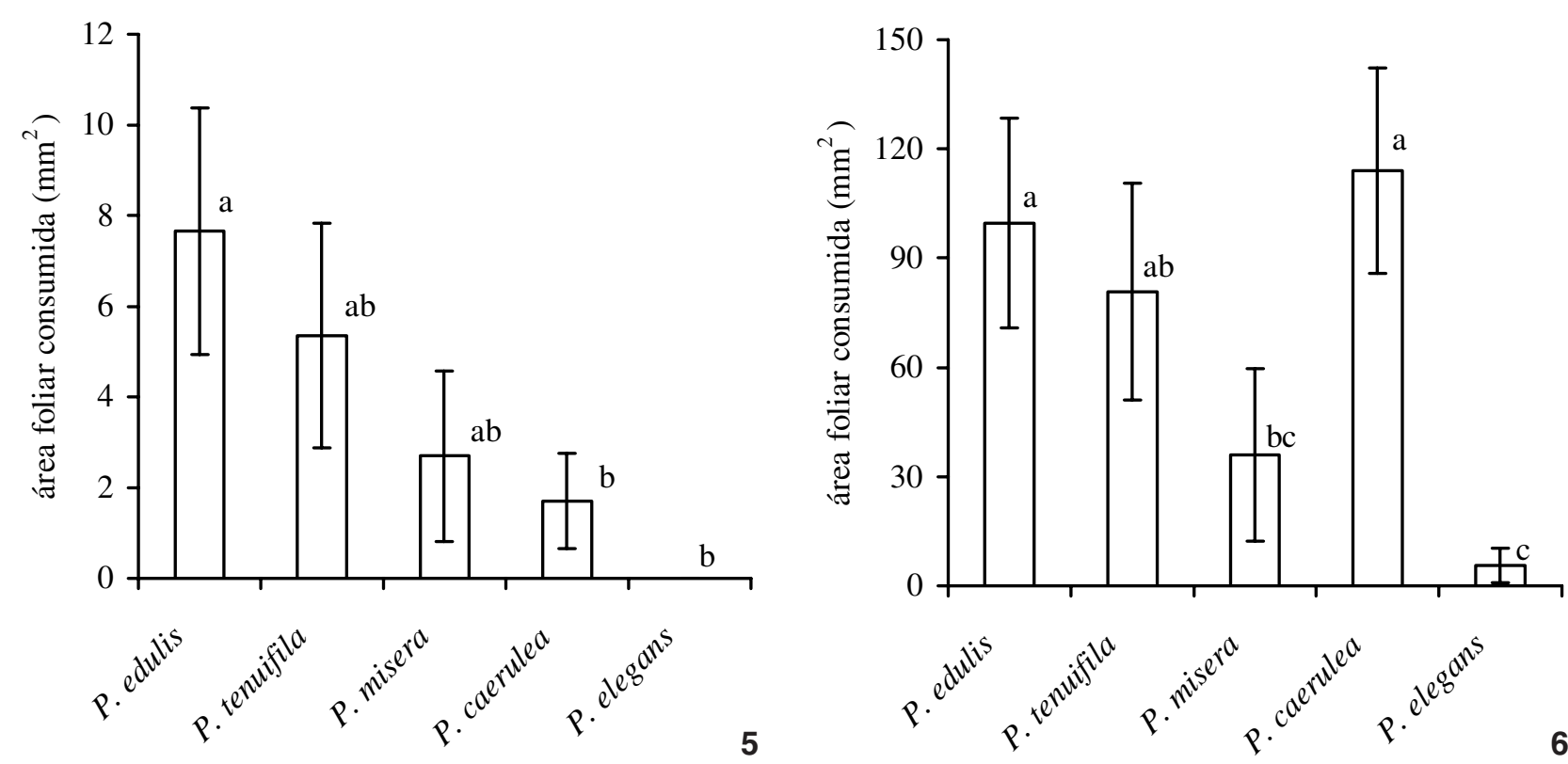

Figuras 5-6. Consumo foliar (média \pm erro padrão) em teste com chance de escolha para larvas de (5) primeiro e (6) quinto instar de $D$. juno juno, em relação a diferentes passifloráceas. Colunas seguidas de letras distintas diferem significativamente (Anovas, um fator, $\mathrm{F}=$ 2,826, $p=0,023$, para o primeiro instar e $F=4,554$ para o quinto instar; testes múltiplos de Tukey, $\alpha=0,05$ ).
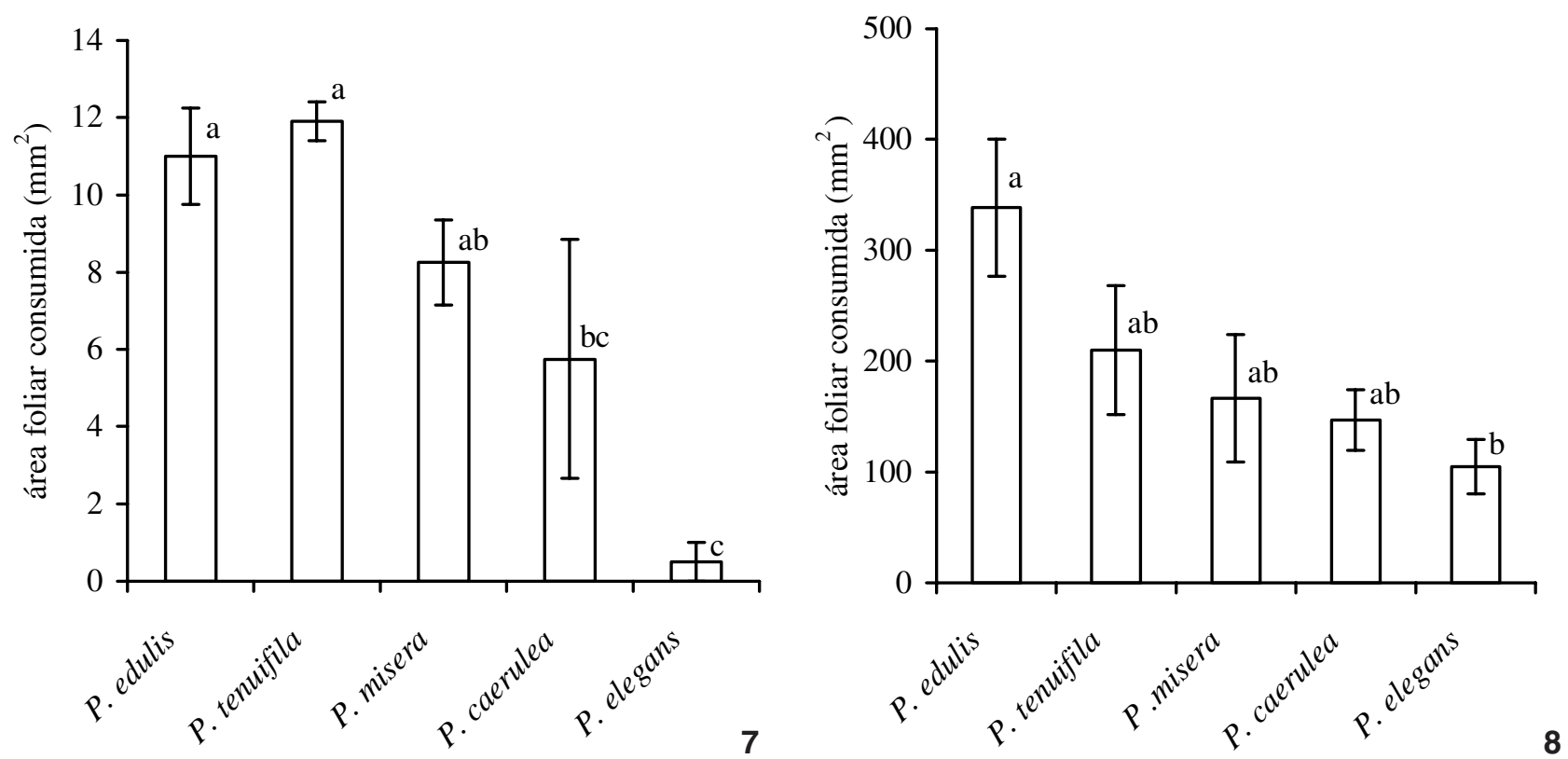

Figuras 7-8. Consumo foliar (média \pm erro padrão) em teste sem chance de escolha para larvas de (7) primeiro e (8) quinto instar de $D$. juno juno, em relação a diferentes passifloráceas. Colunas seguidas de letras distintas diferem significativamente (teste de Kruskal-Wallis, $\mathrm{KW}=36,323, \mathrm{p}=0.0001$; testes múltiplos de Dunn, $\alpha=0,05$, para o primeiro instar; Anova, um fator, $\mathrm{F}=3,368, \mathrm{p}=0,0127$, testes múltiplos de Tukey, $\alpha=0,05$, para o quinto instar).

so de exploração deste grupo de plantas, as quais apresentam, muitas vezes, variação acentuada na distribuição geográfica e fenologia distinta. Isto pode aumentar a gama de plantas para a oviposição em um dado local, garantindo a sobrevivência dos indivíduos principalmente quando da escassez de recursos (Chew 1975, Rodrigues \& Moreira 2002, 2004). 

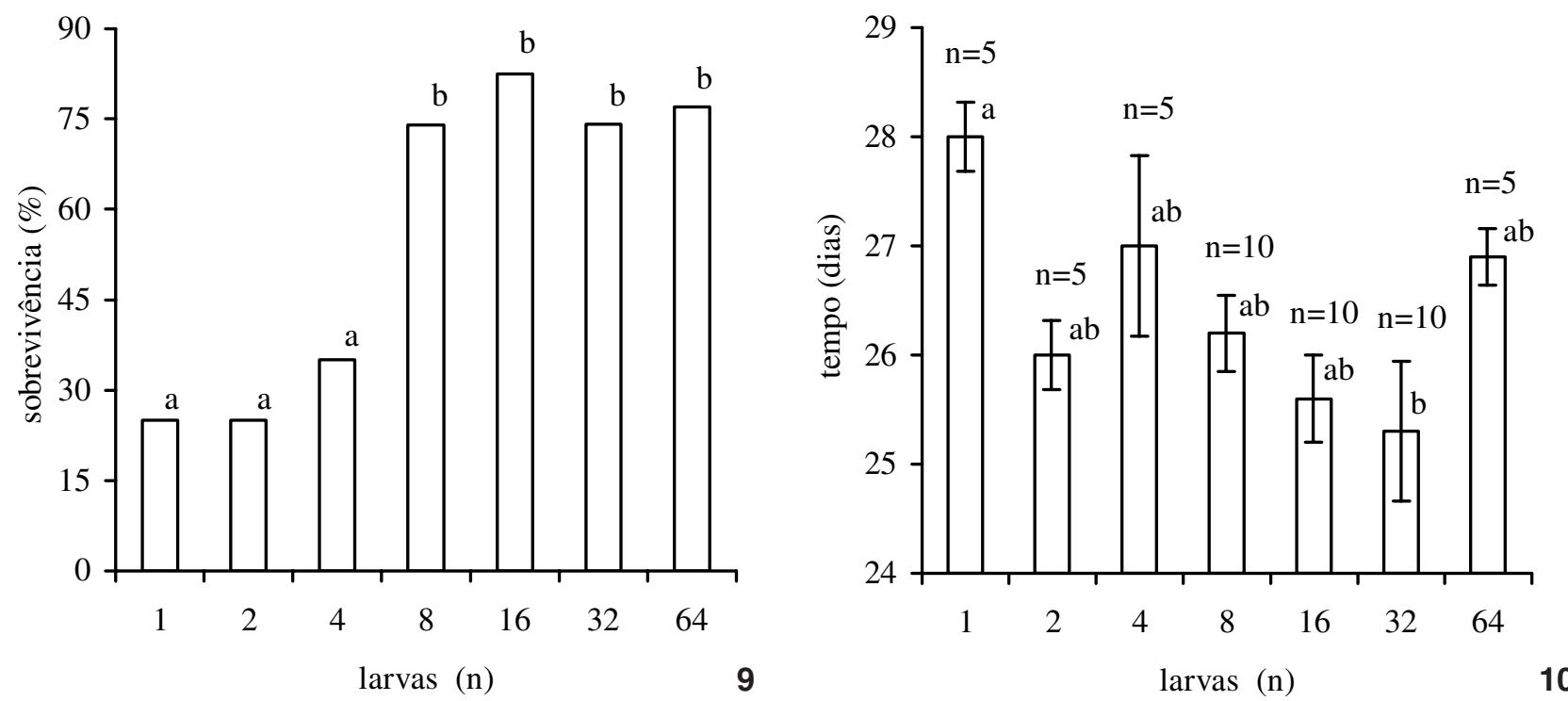

Figuras 9-10. (9) Sobrevivência de $D$. juno juno em relação a larvas foram criadas em diferentes densidades. Colunas seguidas de letras distintas diferem significativamente (testes exatos de Fisher, com correção de Bonferroni, $\alpha=0,023)$; $(10)$ Tempo de desenvolvimento (média \pm erro padrão) dos imaturos de $D$. juno juno, quando criados em diferentes densidades larvais. Colunas seguidas de letras distintas diferem significativamente (Anova, um fator, $F=2,784, p=0,0218$; testes múltiplos de Tukey, $\alpha=0,05$ ).

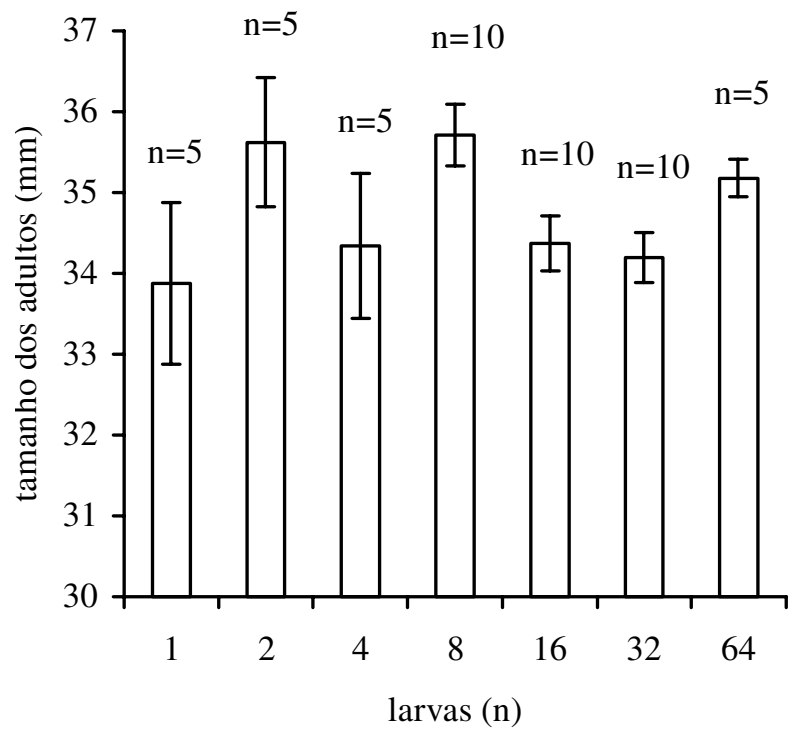

Figura 11. Tamanho dos adultos (média \pm erro padrão) de $D$. juno juno, quando as larvas foram criadas em diferentes densidades. Não há diferença significativa entre as densidades (Anova, um fator, $F=1,673, p=0,1498)$.

O menor tempo para atingir a fase adulta ocorreu em $P$. misera, comparando ao desenvolvimento de larvas de $H$. erato phyllis alimentadas com $P$. suberosa. Assim, deste ponto de vista, podemos inferir que $P$. misera confere maior valor adaptativo, pois o desenvolvimento mais rápido potencialmente reduz o tempo de exposição das larvas aos predadores (CHEw 1975, PRICE et al. 1980). O maior tamanho dos adultos como ocorreu com larvas alimentadas em $P$. misera e $P$. edulis, também pode ser vantajoso, pois, asas maiores podem ampliar a autonomia de vôo (Courtney 1981) influenciando sua sobrevivência, bem como outras atividades biológicas importantes e, especificamente para as fêmeas, onde o tamanho tem sido correlacionado à fecundidade (Courtney 1981, Rodrigues \& Moreira 2002).

Os estudos realizados por LARA et al. (1999) foram os pioneiros no que diz respeito à preferência alimentar de $D$. juno juno, em relação a $P$. edulis, frente a outras passifloráceas do Estado de São Paulo. Os resultados destes pesquisadores indicam preferência alimentar deste lepidoptero por $P$. edulis ou híbridos com esta espécie, tanto nos testes com discos foliares como naqueles com uso de extratos aquosos das passifloráceas. No presente trabalho, $D$. juno juno demonstrou certa preferência por $P$. edulis, mas não rejeitou $P$. misera, $P$. tenuifila e $P$. caerulea, no primeiro ínstar. Assim, pode-se supor que este inseto tem poucas restrições quanto à preferência alimentar, podendo usar outras passifloráceas quando $P$. edulis está ausente.

Os resultados deste trabalho mostram que a sobrevivência dos imaturos de $D$. juno juno foi afetada pela densidade larval. A maior mortalidade ocorreu nos tratamentos com menos de oito larvas e principalmente, nos instares iniciais. Assim, podemos inferir que a agregação nos primeiros instares é crucial para sobrevivência de $D$. juno juno, seja pela manutenção da temperatura (STAMP 1980, BRYANT et al. 2000) ou pela superação das barreiras físicas da planta (FitzGerald 1993, Dussourd \&

Revista Brasileira de Zoologia 22 (1): 43-50, março 2005 
Denno 1991), pois estes insetos foram criados em ambientes controlados e sem predadores. As diferenças no tempo de desenvolvimento em diferentes densidades não foram relevantes, contrastando com o resultado de Borça Jr. et al. (1999) que observaram diferenças neste parâmetro em relação à densidade. O tamanho dos adultos não foi afetado pela densidade larval desta espécie, como observado por Borça Jr. et al. (1999). Podemos supor que o hábito gregário afeta a larva apenas no inicio do desenvolvimento, mas as razões deste efeito ainda não foram identificadas.

Resultados obtidos neste estudo têm implicações tanto do ponto de vista da ecologia evolutiva quanto para o manejo de $D$. juno juno em pomares comerciais de $P$. edulis no RS. Podese inferir que as demais passifloráceas aqui testadas, e que conferiram sobrevivência a este lepidóptero, sejam somente utilizadas de forma ocasional como hospedeira, na ausência ou baixa disponibilidade de plantas de $P$. edulis.

Ou seja, embora a alimentação a base de $P$. misera confira menor tempo de desenvolvimento e maior tamanho a D. juno juno, é pouco provável que esta espécie seja utilizada por ela de forma expressiva. Levantamentos preliminares apontam para o uso de outras passifloráceas, incluindo P. misera. Porém, $P$. edulis é a hospedeira favorita para oviposição por $D$. juno juno no Parque Estadual do Turvo, Porto Alegre e arredores (G. L. Garcias, dados não publicados). Posturas e populações larvais de D. juno juno são encontradas com freqüência em diversas partes do RS em plantas isoladas de $P$. edulis e, principalmente, em pomares comerciais. Observações ao longo de dez anos de extensivos estudos dos herbívoros dos maracujás na região da Depressão Central do RS, onde sabidamente P. misera é abundante (C. Mondin, comunicação pessoal), dão suporte a tal afirmação. Embora não tenhamos dados a respeito, é de se supor que as plantas de $P$. misera, sendo de porte pequeno, não teriam uma massa foliar grande o suficiente para suportar números elevados de larvas de D. juno juno, levando-se em conta seu hábito de oviposição em massa, associado à agregação larval. Aliado a isso, esta passiflorácea encontra-se freqüentemente sujeita a intensa desfolha por outros heliconíneos, como por exemplo H. erato phyllis e Dryas iulia alcionea (Cramer, 1779), que apresentam preferência por P. misera (Perico \& Araujo 1991, Perico 1995, Rodrigues \& Moreira 2002, Kerpel \& Moreira, no prelo).

Embora P. caerulea seja uma planta de tamanho considerável, encontrada com freqüência principalmente nas regiões campestres do RS, não temos indicativos de que seja utilizada freqüentemente por D. juno juno, mas sim por Agraulis vanillae maculosa (Stichel, 1907), outro heliconíneo, comum na região, porém de hábito larval solitário (Perico 1995, G.L. Garcias, dados não publicados, Dell'Erba et al., no prelo). Plantas de $P$. tenuifila possuem uma massa foliar considerável e apresentam ampla distribuição geográfica, porém com densidades acentuadamente baixas nas áreas de ocorrência (SACCo 1980). P. actinia, a qual é encontrada com relativa frequência no nordeste do RS (C. Mondin, comunicação pessoal), corresponde a única espécie citada como hospedeira de D. juno juno no Estado, não estudada no presente trabalho. Entretanto, alimentadas com essa passiflorácea, as larvas de $D$. juno juno sofrem alta mortalidade e retardam o desenvolvimento, comparado a P. edulis (Borça Jr. et al. 1999).

Tomando-se como base os dados obtidos, o uso potencial de espécies nativas de maracujás como hospedeiras alternativas de $D$. juno juno, seja eliminando tais plantas, nas bordas dos pomares, com vistas a reduzir suas populações ou ainda utilizando-as como plantas armadilhas, visando o controle localizado, terá limitado sucesso no contexto prático do manejo desta praga no RS. Porém, testes de escolha quanto a preferência de oviposição de $D$. juno juno, tanto em condições de insetário quanto em campo, necessitam ser realizados frente a tais espécies de maracujá.

\section{AGRADECIMENTOS}

À administração da Estação Experimental Agronômica da UFRGS, Eldorado do Sul, em especial a Paulo Vitor Dutra de Souza, pelo apoio logístico em relação ao cultivo comercial de Passiflora edulis. Sidia M.Callegari-Jacques (UFRGS) prestou assistência em relação aos testes estatísticos. Lucas Kaminski, Elisete Gauer e Geraldo Soares (UFRGS) efetuaram a leitura crítica das primeiras versões do manuscrito. A versão final deste foi grandemente beneficiada por sugestões de André Victor L. Freitas (UNICAMP) e Mirna M. Casagrande (UFPR). Agradecemos também à CAPES pela Bolsa de Doutorado concedida à V. Bianchi, junto ao Programa de Pós-graduação em Ecologia, UFRGS.

\section{REFERÊNCIAS BIBLIOGRÁFICAS}

Andrade, C.S.F. \& M.E.M. НAвib. 1984. Natural occurrence of baculoviruses in populations of some Heliconiini (Lepidoptera: Nymphalidae) with symptomatological notes. Revista Brasileira de Zoologia, Curitiba, 2: 55-62.

Benson, W.W.; K.S. Brown Jr. \& L.E. Gilbert. 1976. Coevolution of plants and herbivores: passion flower butterflies. Evolution, Bolder, 29: 659-680.

Biezanko, C.M. 1949. Acraeidae, Heliconiidae et Nymphalidae de Pelotas e seus arredores (Contribuição ao conhecimento da fisiografia do Rio Grande do Sul). Pelotas, Livraria do Globo,16p.

Boiça, Jr., A.L.; F.M. Lara \& J.C. Oliveira. 1999. Efeito de genótipos de maracujazeiro (Passiflora spp.) e da densidade larval na biologia de Dione juno juno (Cramer) (Lepidoptera: Nymphalidae). Anais da Sociedade Entomológica do Brasil, Londrina, 28: 41-47.

Brown Jr., K.S. 1981. The biology of Heliconius and related genera. Annual Review of Entomology, Stanford, 26: 427456.

BRown JR., K.S. \& O.H.H. MielKe. 1972. The heliconians of Brazil (Lepidoptera: Nymphalidae). Part. II. Introduction and general comments, with a supplementary revision of the tribe. Zoologica, New York, 57: 1-40.

Revista Brasileira de Zoologia 22 (1): 43-50, março 2005 
BRYANT, S.R.; C.D. Thomas \& J.S. BaLe. 2000. Thermal ecology of gregarious and solitary nettle-feeding nymphalid butterfly larvae. Oecologia, Berlin, 122: 1-10.

CHew, F.S. 1975. Coevolution of pierid butterflis and their cruciferous foodplants. I. The relative quality of available resources. Oecologia, Berlin, 20: 117-127.

Clark, B.R. \& S.H. Faeth. 1997. The consequences of larval aggregation in the butterfly Chlosyne lacinia. Ecological Entomology, London, 22: 408-415.

Conover, W.J. 1980. Pratical nonparametric statistics. New York, John Wiley and Sons, $2^{\text {nd }}$ ed., 493p.

CourTney, S.P. 1981. Coevolution of pierid butterflies and their cruciferous foodplants. III. Anthocaris cardamines (L.) survival, development and oviposition on different hostplants. Oecologia, Berlin, 51: 91-96.

Dell'Erba, R.; L.A. Kaminski \& G.R.P. Moreira (no prelo). O estágio de ovo dos Heliconiini (Lepidoptera, Nymphalidae) do Rio Grande do Sul, Brasil. Iheringia, Série Zoologia, Porto Alegre.

Denno, R.F. \& B. Benrey. 1997. Aggregation facilitates larval growth in the neotropical nymphalid butterfly Chlosyne janais. Ecological Entomology, London, 22: 133-141.

Dussourd, D.E. \& R.F. Denno. 1991. Deactivation of plant defense: correspondence between insect behavior and secretory canal architecture. Ecology, Templeton, 72: 1383-1397.

FitzGERALD, T.D. 1993. Sociality in caterpillars, p. 373-403. In: N.E. STAMP \& T.M. CASEY (Eds). Caterpillars: ecological and evolutionary constraints on foraging. New York, Chapman \& Hall, XIII+587p.

HANSON, F.E. 1983. The behavioral and neurophysiological basis of food plant selection by lepidopterous larvae, p. 3-23. In: S. AHMAD (Ed.). Herbivorous insects: host-seeking behavior and mecanisms. New York, Academic Press, 257p.

Kerpel, S.M. \& G.R.P. Moreira (no prelo). Absence of learning and local specialization on host plant selection by Heliconius erato. Journal of Insect Behavior, New York.

Lara, F.M.; A.L. BoiçA JR. \& J.C. Barbosa. 1999. Preferência alimentar de Dione juno juno (Cramer) por genótipos de maracujazeiros e avaliação do uso de extratos aquosos. Scientia Agricola, Piracicaba, 56: 665-671.

Lewis, A.C. \& H.F. van EMden. 1986. Assays for insect feeding, p. 95-119. In: J.R. Miller \& T.A. Miller (Eds). Insect-plant interactions. New York, Springer-Verlag, 342p.

LORDELLO, L.G.E. 1956. Insetos que vivem sôbre o maracujazeiro. III - Notas acêrca de Dione juno (Cramer) (Lep., Nymphalidae) e relação de alguns outros insetos habitualmente coligidos de Passiflora spp. Revista de Agricultura, Piracicaba, 29: 23-29.

Menna-Barreto, Y. \& A.M. Araújo. 1985. Evidence for host plant preferences in Heliconius erato phyllis from southen Brazil (Nymphalidae). Journal of Research on the Lepidoptera, Arcadia, 24: 41-46.

Mugrabi-Oliveira, E. \& G.R.P. Moreira. 1996. Size of and damage on shoots of Passiflora suberosa (Passifloraceae) influence oviposition site selection of Heliconius erato phyllis (Lepidoptera, Nymphalidae). Revista Brasileira de Zoologia, Curitiba, 13: 939-953.

Muyshondt, A.; A.M. Young \& A. Muyshondt Jr.1973. The biology of the butterfly Dione juno huascama (Nymphalidae: Heliconiinae) in El Salvador. Journal of the New York Entomological Society, New York, 81: 137-151.

Périco, E. 1995. Interação entre quatro espécies de Heliconiini (Lepidoptera: Nymphalidae) e suas plantas hospedeiras (Passifloraceae) em uma floresta secundária do Rio Grande do Sul, Brasil. Biociências, Porto Alegre, 3: 3-18.

Périco, E. \& A.M. Araujo. 1991. Suitability of host plants (Passifloraceae) and their acceptableness by Heliconius erato and Dryas iulia (Lepidoptera; Nymphalidae). Evolución Biológica, Bogotá, 5: 59-74

Price, P.W.; C.E. Bouton; P. Gross; B.A. McPheron, J.N. Thompson \& A.E. WeIs. 1980. Interactions among three trophic levels: influence of plants on interactions between insect herbivores and natural enemies. Annual Review of Ecology and Systematics, Stanford, 11: 41-65.

Rodrigues, D. \& G.R.P. Moreira. 1999. Feeding preference of Heliconius erato (Lep.: Nymphalidae) in relation to leaf age and consequences for larval performance. Journal of the Lepidopterists' Society, Los Angeles, 53: 108-113.

Rodrigues, D. \& G.R.P. Moreira. 2002. Geographical variation in larval host-plant use by Heliconius erato (Lepidoptera: Nymphalidae) and consequences for adult life history. Brazilian Journal of Biology, São Carlos, 62: 321-332.

RodRIGUES, D. \& G.R.P. MoReIRA. 2004. Seasonal variation in larval host-plants and consequences for Heliconius erato (Lepidoptera: Nymphalidae) adult body size. Austral Ecology, Carlton, 29: 437-445.

SACco, J.C. 1980. Passifloráceas, p.1-132. In: R. Reitz (Ed.). Flora ilustrada catarinense. Itajaí, Herbário Barbosa Rodrigues, $132 p$.

STAMP, N.E. 1980. Egg deposition patterns in butterflies: why do some species cluster their eggs rather than deposit them singly? The American Naturalist, Chicago, 115: 367-380.

SLANSKY, F. JR. 1993. Nutritional ecology: the fundamental quest for nutrients, p.29-91. In: N.E. Stamp \& T.M. CASEY (Eds). Caterpillars: ecological and evolutionary constraints on foraging. New York, Chapman \& Hall, XIII+587p.

TAVARES, M.; L.A. Kaminski \& G.R.P. MoReIra. 2002. Morfologia externa dos estágios imaturos de heliconíneos neotropicais. II. Dione juno juno (Cramer) (Lepidoptera, Nymphalidae, Heliconiinae). Revista Brasileira de Zoologia, Curitiba, 19: 961-976.

Vanderplank, J. 1991. Passion flowers and passion fruit. Cambridge, MIT, 176p.

ZAR, J.H. 1999. Biostatistical analysis. New Jersey, PrenticeHall, $4^{\text {th }}$ ed., XII+663p.

Recebido em 13.V.2004; aceito em 05.I.2005. 